\title{
Impact of the high baseline anti-A/B antibody titer on the clinical outcomes in ABO-incompatible living donor liver transplantation
}

\author{
Boram Lee ${ }^{1}$, Jai Young Cho ${ }^{1}$, Suk Kyun Hong ${ }^{2}$, YoungRok Choi ${ }^{2}$, Hae Won Lee ${ }^{1}$, Namjoon Yi ${ }^{2}$, Kwang-Woong Lee ${ }^{2}$, \\ Kyung-Suk Suh ${ }^{2}$, Ho-Seong $\operatorname{Han}^{1}$
}

\footnotetext{
${ }^{1}$ Department of Surgery-Hepatobiliary, Seoul National University Bundang Hospital, Seongnam, Korea

${ }^{2}$ Department of Surgery-Transplantation, Seoul National University Hospital, Seoul, Korea
}

Background: Recently, advances in desensitization protocol have made $A B O$-incompatible (ABOi) living donor liver transplantation (LDLT) feasible option in the era of organ shortage. Although, multiple sessions of plasmapheresis can successfully reduce preformed anti-A/B titer prior to transplantation, the clinical significance of baseline anti-A/B antibody titers remains uncertain. The aim of this study is to investigate the clinical outcomes of ABOi LDLT in patients with a high baseline anti-A/B antibody titer. Methods: A total of 50 patients who received ABOi LDLT from 2010 to 2020 at two tertiary hospitals were evaluated retrospectively. Two centers used a protocol composed of rituximab, plasmapheresis, and/or splenectomy. The patients were classified according to baseline anti-A/B titer $(<1: 256, n=88$ or $\geq 1: 256, n=62)$ and compared the clinical outcomes among these groups. Graft survival rates were calculated using the Kaplan-Meier methods according to the groups.

Results: In the high baseline titer group, the number of plasmaphereses required to reach the target titer (1:16) was significantly higher ( $4.4 \pm 2.2$ sessions) than in the low baseline titer group $(1.9 \pm 1.2$ sessions, $P<0.001)$. Fourteen patients $(16.4 \%)$ in high baseline titer group and seven patients $(9.2 \%)$ in low baseline titer group experienced postoperative titer rebound to $\geq 1: 32,(P=0.014)$. The occurrence of both cellular rejection and antibody-mediated rejection did not show a significant difference $(P=0.251$ and $\mathrm{P}=0.147$, respectively). The 1-, 3-, and 5-year graft survival was not different among groups (high titer vs. low titer; $94.2 \%, 83.3 \%$, and $59.0 \%$ vs. $92.1 \%, 86.3 \%$, and $79.5 \% ; P=0.326$ ). In multivariate analysis showed that high baseline anti-A/B titer and postoperative rebound titer did not adversely affect clinical outcomes after ABOi LDLT.

Conclusions: Although, the patients with high baseline anti-A/B titer showed the higher tendency of postoperative antibody rebound, the baseline and rebound anti-A/B titer may not be as important factors for clinical outcomes of ABOi LDLT if appropriate desensitization is performed.

Corresponding author: Jai Young Cho

E-mail: jychogs@gmail.com

(C) The Korean Society for Transplantation

This is an Open Access article distributed under the terms of the Creative Commons Attribution Non-Commercial License (http://creativecommons.org/licenses/by-nc/4.0/) which permits unrestricted non-commercial use, distribution, and reproduction in any medium, provided the original work is properly cited. 